\title{
New evidence in oncologic and cardiocirculatory medicine
}

\author{
Andrea Alberto Conti · Beatrice Dilaghi · \\ Pietro Amedeo Modesti · Carlo Nozzoli
}

Published online: 13 December 2008

(C) SIMI 2008

\section{Comparison between the therapy with melphalan- prednisone alone and associated with bortezomib in the initial treatment of multiple myeloma}

The combined therapy with bortezomib, melphalan and prednisone proved to be more effective than the therapy composed by melphalan and prednisone alone in the treatment of patients affected by recently diagnosed multiple myeloma not candidate to standard therapy at the maximum dosage and to the transplantation of stem cells.

A group of international researchers conducted a randomised, phase 3 "open label" study in which 682 symptomatic patients were enrolled with a new diagnosis of multiple myeloma, who could not be treated with maximum therapy and stem cells transplantation because of their age ( $\geq 65$ years) or because of the presence of concomitant diseases. Aim of this study was the comparison of the effectiveness of the treatment with melphalan and prednisone alone with that with the same drugs associated with bortezomib. The enrolled patients were randomised to perform nine cycles of therapy of 6 weeks each during which they assumed melphalan at the dosage

A. A. Conti $(\bowtie) \cdot$ P. A. Modesti

Department of Critical Care Medicine and Surgery,

University of Florence and Don Carlo Gnocchi Foundation,

IRCCS Florence, Viale Morgagni 85, 50134 Florence, Italy

e-mail: aa.conti@dac.unifi.it

B. Dilaghi

Department of Emergency Medicine,

Azienda Ospedaliero-Universitaria Careggi, Florence, Italy

C. Nozzoli

General Medicine Unit,

Azienda Ospedaliero-Universitaria Careggi,

Florence, Italy of $9 \mathrm{mg} / \mathrm{m}^{2}$ of body area, and prednisone $\left(60 \mathrm{mg} / \mathrm{m}^{2}\right)$ in days from 1 to 4 , alone or in combination with bortezomib $\left(1.3 \mathrm{mg} / \mathrm{m}^{2}\right)$ in days $1,4,8,11,22,25,29$ and 32 during the first 4 cycles and in days 1, 8, 22 and 29 during the other 5 cycles. The major end point was represented by the time to disease progression; secondary end points included the percentage of complete response, the length of the response and total survival.

The results of this study show that the time to the progression of the disease in patients receiving bortezomib, melphalan and prednisone (bortezomib group) was 24 months, if compared to 16.6 months of the patients receiving melphalan and prednisone (control group). The risk ratio for the bortezomib group was 0.48 $(P<0.001)$. The percentage of patients who had an at least partial response was $71 \%$ in the bortezomib group and $35 \%$ in the control group; a complete response was recorded in 30 and $4 \%$, respectively $(P<0.001)$. The median length of the response was 19.9 months in the first group and 13.1 months in the second one. The risk ratio for total survival was 0.61 for bortezomib group $(P=0.008)$.

The combined therapy with bortezomib, melphalan and prednisone is more effective than the therapy with melphalan and prednisone alone in patients affected by recently diagnosed multiple myeloma who cannot undergo standard therapy at the maximum dosage and stem cells transplantation.

\section{Reference}

San Miguel JF et al (2008) Bortezomib plus melphalan and prednisone for initial treatment of multiple myeloma. N Engl J Med 359:906-917 
The performance of a single reader adopting a computer-aided detection system can be an appropriate alternative to double reading in identifying breast cancer by means of mammography

The percentage of breast cancers identified by double reading is comparable to the proportion of tumours detected by single reading with computer-aided detection in an equivalence trial in which more than 31,000 women were evaluated.

A group of British researchers designed and conducted a trial to assess the diagnostic sensitivity of screening mammograms analysed by single readers with the support of a computer-aided detection system compared to the sensitivity of double reading. 31,057 women, undergoing regular screening, were enrolled. They were randomly allocated to double reading, single reading with computeraided identification or both at a ratio of $1: 1: 28$. The first group was composed by 1,000 subjects, the second was made up of 1,000 individuals and the third one included 28,000 women. The major end point was constituted by the percentage of breast tumours identified on the basis of the different diagnostic management strategies, and by the rate of recall in the third group. The results of this study show that the proportion of breast cancers diagnosed was 199 out of $227(87.7 \%)$ with double reading and 198 out of 227 $(87.2 \%)$ with single reading supported by the computeraided detection $(P=0.89)$. The rate of recall was $3.4 \%$ in the double reading group and $3.9 \%$ in the single reading with computer-aided detection group; the difference of these two rates was significant $(P<0.001)$. Sensitivity, specificity and positive predictive value for single reading with the support of the computer-aided system were 87.2, 96.9 and $18 \%$. Sensitivity, specificity and positive predictive value for double reading were $87.7,97.4$ and $21.1 \%$.

The authors conclude that the reading of a screening mammography by a single operator with a computer-aided identification system can improve the proportion of detection of breast tumours if compared to single reading alone, and could represent an appropriate alternative to double reading.

\section{Reference}

Gilbert FJ et al (2008) Single reading with computer-aided detection for screening mammography. N Engl J Med 359:1675-1684

\section{Alteplase improves clinical outcomes in patients with acute ischemic stroke also if administered 3-4.5 $\mathrm{h}$ after the insurgence of symptoms}

The results of a randomised controlled clinical trial, conducted on more than 800 patients, indicate that the thrombolytic alteplase, administered i.v. 3-4.5 $h$ after the beginning of clinical symptoms, improves in a significant way the clinical results of treated patients.

At present alteplase administered i.v. is the only available and effective medical therapy for the treatment of acute ischemic stroke. Around mid 1990s the results of the National Institute of Neurological Disorders and Stroke (NINDS) study showed the effectiveness of this drug administered within $3 \mathrm{~h}$ from the insurgence of clinical symptoms. The researchers of the European Cooperative Acute Stroke Study (ECASS) assessed the effectiveness and safety of the i.v. administration of alteplase after $3 \mathrm{~h}$ from the beginning of symptoms and precisely 3-4.5 h after the onset of stroke symptoms. In this multicentric study, from which subjects with cerebral haemorrhage and large cerebral infarction were excluded, 821 patients were enrolled; they were randomly assigned to alteplase (418) or to placebo (403). Median alteplase administration time was $3 \mathrm{~h}$ and $59 \mathrm{~min}$. A total of $52.4 \%$ of patients treated showed a favourable outcome, if compared to $45.2 \%$ individuals in the placebo group (odds ratio: 1.34, with a $95 \%$ confidence interval included between 1.02 and 1.76; $P=0.04)$. Mortality was not significantly different in the two groups, while the incidence of intracranial haemorrhage resulted higher in patients undergoing alteplase (27\%) if compared to placebo group individuals $(17.5 \%)$ $(P=0.001)$.

The treatment with alteplase, administered between 3 and $4.5 \mathrm{~h}$ after the insurgence of stroke symptoms, even if it is associated with a significantly higher incidence of intracranial haemorrhages, improves in a significant way the clinical results of treated patients.

\section{Reference}

Hacke W et al (2008) Thrombolysis with alteplase 3 to 4.5 hours after acute ischemic stroke. N Engl J Med 359:1317-1329

\section{Cardiovascular risk assessment in England and Wales}

The addition to classical risk factors of elements such as the ethnic group, the socio-economic status and the specific definition of clinical conditions improves the accuracy of the evaluation of cardiovascular risk in a sample of the British population including more than two millions subjects.

Different tools for the assessment of the cardiovascular risk are available today, but, since they usually do not fully consider the influence of the ethnic group and of the social status, the overall definition of cardiovascular risk is likely to result underestimated, in particular in 
high risk groups. This British study had as its major end point the derivation and the validation of QRISK2, an algorithm for the evaluation of cardiovascular risk also considering the ethnic group, socio-economic variables and specific clinical conditions. The researchers have therefore evaluated, in a prospective cohort study (with data collected from general medicine between 1993 and 2008), 2.3 millions of subjects of age included between 35 and 74 years with 140,000 cardiovascular events. The results of the statistical analysis of this new tool indicate that QRISK2 has a better evaluation and discriminatory capacity than Framingham score. On the clinical-epidemiological ground, the use of QRISK2 showed that in the South-East Asian population the risk of cardiovascular disease is significantly higher if compared to reference Caucasian populations, and the risk is independent of family history, diabetes mellitus and social discomfort. The population identified by QRISK2 results, with regard to the risk threshold of $20 \%$ at 10 years (the one recommended for the administration of statins in primary cardiovascular prevention), was at higher cardiovascular risk than that identified by the modified Framingham tool.

The incorporation of the ethnic group and of the socioeconomic status therefore improves the accuracy in the definition of subjects at high cardiovascular risk in a large sample of population representative of the British reality.

\section{Reference}

Hippisley-Cox J et al (2008) Predicting cardiovascular risk in England and Wales: prospective derivation and validation of QRISK2. BMJ 336:1475-1482

\section{Drug-eluting stent implantation is associated with a reduction in the mortality at two years in comparison with bare-metal stent implantation in more than 7,000 patients with acute myocardial infarction}

The analysis of the US researchers conducting this study indicate that in patients with myocardial infarction drugeluting stents, if compared to bare-metal stents, are associated with a decrease in 2-year mortality and with a reduction of the need for repeat revascularizations.

Percutaneous coronary interventions (PCI) with stents constitute the first-line treatment for patients suffering acute myocardial infarction who can access in short time an equipped cath lab led by expert health staff. So far scientific comparisons studies between PCI with drug-eluting stents and PCI with bare-metal stents are of limited dimensions and length. The authors of this study have therefore conducted their survey by performing a propensity-score matching and propensity-score analyses on the basis of the Massachusetts Data Analysis Center database, and by identifying and re-analysing every adult already undergone PCI with stent implantation between 1 April 2003 and 30 September 2004 in acute care non-federal hospitals in Massachusetts. The studied subjects were assigned to the group of drug-eluting stents or to the baremetal stents group; patients with the implantation of both drug-eluting and bare-metal stents were excluded from this survey.

More than 4,000 $(4,016)$ patients treated because of acute myocardial infarction implanted with drug-eluting stents and over 3,000 $(3,201)$ subjects implanted with baremetal stents were considered. The mortality rate at two years, adjusted by risk, was $10.7 \%$ in the drug-eluting stents group and $12.8 \%$ in the bare-metal stents group $(P=0.02)$. The need for new revascularization procedures was significantly different between the drug-eluting stents group $(9.6 \%)$ and the bare-metal stents group $(14.5 \%)$ $(P<0.001)$.

The authors of this analysis conclude that, in patients with acute myocardial infarction, the application of drugeluting stents, if compared to bare-metal stents, is associated with a significant reduction of the mortality at 2 years and with a significant decrease in the need for new revascularization of target coronary vessels.

\section{Reference}

Mauri L et al (2008) Drug-eluting or bare-metal stents for acute myocardial infarction. N Engl J Med 359:1330-1342

Conflict of interest statement The authors declare that they have no conflict of interest related to the publication of this manuscript. 